\title{
Aplicación metodológica: the integrated use of management system standards para la integración de sistemas de gestión*
}

\author{
Paula Alejandra Moreno Parra ${ }^{* *}$ \\ Byron Restrepo Benavidez ${ }^{* * *}$ \\ Paola Alejandra Sánchez Martín ${ }^{* * * *}$
}

Recibido: 16 de junio de 2020

Revisado: 1 de marzo de 2021

Aceptado: 13 de agosto de 2021

${ }^{*}$ Articulo de resultado de investigación.
** Bacterióloga y laboratorista clínica, especialista en Gerencia en Salud Ocupacional, especialista
en Auditoria en Salud, especialista en Gerencia y Administración de Calidad y máster en Ciencias
en Calidad y Gestión Integral por la Universidad Santo Tomás convenio Icontec, Bogotá,
Colombia. Correo electrónico: paula.morenop@usantotomas.edu.co, paulaleja@gmail.com;
ORCID: https://orcid.org/0000-0002-8200-3357; CvLAC:
https://scienti.minciencias.gov.co/cvlac/visualizador/generarCurriculoCv.do?cod rh=0001009451
Ci** Microbiólogo industrial, especialista en Gerencia y Administración de Calidad y máster en
Colombias en Calidad y Gestión Integral por la Universidad Santo Tomás convenio Icontec, Bogotá,
ORCID: https://orcid.org/0000-0002-3839-9702; CVLAC:
https://scienti.minciencias.gov.co/cvlac/visualizador/generarCurriculoCv.do?cod rh=0001853601
Ci*** Ingeniería biomédica, especialista en Gerencia y Administración de Calidad y máster en
Colombias en Calidad y Gestión Integral por la Universidad Santo Tomás convenio Icontec, Bogotá,
ORCID: https://orcid.org/0000-0002-3694-3970; CVLAC:
https://scienti.minciencias.gov.co/cvlac/visualizador/generarCurriculoCv.do?cod rh=0001853555

Signos, Investigación en Sistemas de Gestión

ISSN: 2145-1389 | e-ISSN: 2463-1140 | DOI: https://doi.org/10.15332/24631140

Vol. 13 N.0 2 | julio-diciembre de 2021 
Citar como:

Moreno Parra, P. A., Restrepo Benavidez, B. y Sánchez Martínez, P. A. (2021). Aplicación metodológica: the integrated use of management system standards para la integración de sistemas de gestión. Signos, Investigación en Sistemas de Gestión, 13(2). https://doi.org/10.15332/24631140.6668

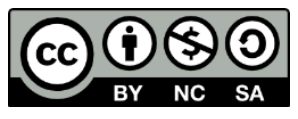

\section{Resumen}

Diversas metodologías se han desarrollado desde los años noventa para la integración de múltiples sistemas de gestión en todo tipo de organización. Sin embargo, en la actualidad es poco conocido que la International Standard Organization (ISO) ha desarrollado una metodología de integración propia que permite alinear diferentes perspectivas normativas en una sola gestión. El manual The Integrated Use of Management System Standard (IUMSS) establece un método de cuatro etapas principales (preparación, conexión, incorporación y mantenimiento) en las cuales la estructura de alto nivel sirve como guía para integrar requisitos comunes, homólogos y abordar los específicos. El objetivo de la investigación fue establecer una metodología de integración de las normas: NTC ISO 14001: 2015 sistema de gestión ambiental, NTC ISO 45001:2018 sistema de seguridad y salud en el trabajo y NTC ISO 13485:2016 sistemas de gestión de calidad para dispositivos médicos; haciendo uso del manual a través de una descripción cualitativa y analítica. Finalmente, se realizó la validación de la metodología de integración de las normas a través del método del ábaco de Régnier, concluyendo que el manual es una herramienta robusta orientada a la disminución del exceso de redundancias y al cierre de brechas entre los requisitos de las normas ISO integradas y los procesos de una organización.

Palabras clave: sistemas integrados de gestión, NTC ISO 14001:2015, NTC ISO 45001:2018, NTC ISO 13485:2016, metodología de integración, brechas. 


\section{Methodological application: the integrated use of management system standards for management systems integration}

\section{Abstract}

Various methodologies have been developed since the 1990 for integrating multiple management systems in all types of organizations. However, at present it is little known that the International Standard Organization (ISO) has developed its own integration methodology that allows aligning different normative perspectives in a single management. The Manual The Integrated Use of Management System Standard (IUMSS) sets out a four main stage method (preparation, connection, incorporation and maintenance) wherein the high-level structure serves as a guide to integrate common requirements, counterparts and to address the specific ones. The research objective was to establish a methodology for the integration of the follwoing standards: NTC ISO 14001: 2015 environmental management system, NTC ISO 45001: 2018 occupational health and safety system and NTC ISO 13485: 2016 quality management systems for medical devices; by using the manual through a qualitative and analytical description. Finally, the standards integration methodology was validated through Régnier's abacus method, concluding that the manual is a robust tool aimed at reducing excess redundancies and closing gaps between the integrated ISO standards and an organization's processes requirements.

Keywords: integrated management systems, NTC ISO 14001: 2015, NTC ISO 45001: 2018, NTC ISO 13485: 2016, integration methodology, gaps. 


\section{Aplicação metodológica: o uso integrado de padrões de sistemas de gestão para integração de sistemas de gestão}

\section{Resumo}

Diversas metodologias foram desenvolvidas desde os anos 90 para integrar múltiplos sistemas de gestão em todos os tipos de organizações. Entretanto, hoje é pouco conhecido o fato da Organização Internacional de Normalização (ISO) ter desenvolvido sua própria metodologia de integração que permite alinhar diferentes perspectivas normativas em uma única gestão. O Manual The Integrated Use of Management System Standard (IUMSS) estabelece um método em quatro etapas principais (preparação, conexão, incorporação e manutenção) no que a estrutura de alto nível serve como um guia para integrar requisitos comuns, equivalentes e específicos. O pesquisa visou estabelecer uma metodologia de integração dos padroes: NTC ISO 14001: sistema de gestão ambiental 2015, NTC ISO 45001:2018 sistema de segurança e saúde ocupacional e NTC ISO 13485:2016 sistemas de gestão de qualidade para dispositivos médicos; usando o manual através de uma descrição qualitativa e analítica. Finalmente, a metodologia de integração dos padrões foi validada através do método do ábaco de Régnier, concluindo que o manual é uma ferramenta robusta que visa reduzir as redundâncias excessivas e fechar lacunas entre os requisitos dos padrões ISO integrados e os processos de uma organização.

Palavras-chave: sistemas de gestão integrada, NTC ISO 14001:2015, NTC ISO 45001:2018, NTC ISO 13485:2016, metodologia de integração, lacunas.

\section{Introducción}

En la actualidad todo tipo de organización debe enfrentarse a mercados y contextos cambiantes, lo que obliga a las áreas directivas a fortalecer la estructura administrativa a través de modelos de gestión que sustenten el 
direccionamiento estratégico y promuevan la estabilidad, la buena competencia, la generación de resultados con menos recursos, la reducción de la duplicidad en los procesos, la mitigación de la burocracia y el aumento efectivo del desempeño para impactar positivamente en las necesidades y exigencias de todas las partes interesadas (Pullés y Bataller Venta, 2016), (Cabrera et ál., 2015).

$\mathrm{Al}$ considerar los beneficios que han alcanzado ciertas organizaciones al implementar diversos sistemas de gestión, varias investigaciones se han realizado sobre la manera en que deben integrarse y por esta razón progresivamente cada año se realizan estudios sobre el tema (Bernardo et ál., 2017). Por un lado, se han descrito las ventajas y las dificultades administrativas del proceso de integración relacionadas con el exceso de burocracia, redundancias, costos, falta de motivación de empleados y directivos para gestionar diferentes sistemas de gestión a la vez (Bernardo et ál., 2015); y, por otra parte, se plantean metodologías facilitadoras que se fundamentan en casos exitosos de varias compañías y sectores que, a través de su experiencia, promueven la integración como factor determinante para alcanzar la eficiencia a través de la optimización del uso de recursos, aumentar la capacidad de satisfacer las necesidades de las partes interesadas, mejorar el clima organizacional, promover el trabajo en equipo, mejorar la comunicación de todas las áreas, intercambiar conocimientos, mejorar la imagen corporativa, entre otros (Moumen y Aoufir, 2017; Bernardo et ál., 2017; Gianni et ál., 2017).

En la integración de sistemas de gestión, se deberá considerar lo descrito por Wilkinson y Dale (1999); y Karapetrovic y Jonker (2003). En sus trabajos se resalta la necesidad de definir y establecer una metodológica genérica o aplicable a todo tipo de organización que permita agrupar los elementos comunes entre los diferentes sistemas a integrar, flexibilidad para satisfacer los requerimientos normativos, compatibilidad con el ciclo 
PHVA y enfoques sistémicos. Además, se debe tener en cuenta que, si otras actividades y operaciones no pueden integrarse, deberán ser rediseñadas de manera que se vuelvan sinérgicas con las funciones que han sido integradas (Nunhes et ál., 2017).

Sin embargo, la existencia de múltiples metodologías integradoras, planteadas por diferentes autores, suscita confusión al momento de discernir sobre cuál de ellas es la indicada para la organización que se encuentre interesada en iniciar un proceso de integración. En el artículo publicado por Heras et ál. (2007) se mencionan todas las investigaciones realizadas entre 1997 y 2006 sobre los elementos que deberían ser abordados al momento de realizar dicho proceso; no obstante, señalan que es difícil pensar en un modelo justo y específico para una organización, teniendo en cuenta que cada proceso es individual y debe ajustarse al entorno organizacional.

En respuesta a la problemática planteada, la ISO ha publicado en el año 2018, en su segunda versión, un manual que plantea una base metodológica que busca integrar los requisitos de diferentes normas en un solo sistema de gestión, de manera efectiva y eficiente a través de un componente teórico y la descripción de casos exitosos en organizaciones de cualquier sector.

El manual fue desarrollado a pedido de la junta de gestión técnica de ISO para las organizaciones que buscan orientación sobre cómo integrar los requisitos de múltiples normas ISO en el sistema de gestión. Desde su primera edición en el año 2008, el IUMSS ha tenido un número sustancial de cambios estos incluyen la introducción de la estructura de alto nivel ISO, proporcionada en las Directivas ISO / IEC Parte 1, Cláusula SL.9. Con la finalidad de poderse adaptar a las normas ISO y también aquellas normas que no pertenecen a la misma familia (ISO, 2018). 
Dado lo anterior, el objetivo del presente artículo es describir la metodología de integración, la cual se deriva del proyecto de investigación titulado Integración de las Normas NTCISO 14001:2015, NTCISO 45001:2018 y NTCISO 13485:2016 y su alineación a un sistema de gestión integral basado en la GTC 180:2008, en el sector de comercialización para dispositivos médicos en Colombia (Moreno, Restrepo, y Sanchez, 2020).

Para ello se realizó la traducción, interpretación y establecimiento de los lineamientos del manual (IUMSS) como metodología de integración para las normas NTC ISO 14001:2015, NTC ISO 45001:2018 y NTC ISO 13485:2016, debido a que en la actualidad se desconoce y no se encuentra referenciado en investigaciones anteriores donde se haya implementado. Por esa razón, resulta de interés para investigadores y organizaciones disponer de un documento que interprete estas directrices oficiales dadas por la ISO para integrar cualquiera de sus normas, respetando el contenido específico de cada una de ellas.

\section{Metodología}

La investigación se realizó bajo un enfoque cualitativo, descriptivo y analítico, cuyo alcance fue proponer la integración de las Normas NTC ISO 14001:2015, NTC ISO 45001:2018 y NTC ISO 13485:2016, teniendo en cuenta la metodología propuesta en el manual IUMSS. El proceso de integración da inicio con la traducción e interpretación del manual, con el objetivo de comprender su estructura para posteriormente desarrollar la propuesta de integración.

Consecutivamente, se realizó el análisis del manual y se evidenció que su diseño y estructura se encuentran dada en tres capítulos. En el capítulo 1 se describen los fundamentos de un sistema de gestión y cómo se relacionan las estrategias, planes y procesos de una organización; en el 
capítulo 2 se describe la estructura y el contenido de diferentes sistemas de gestión normativos y su aplicación; y, en el capítulo 3, se describe cómo una organización puede integrar los requisitos de varias normas dentro de un sistema de gestión. El manual fue estructurado de esta manera para que los usuarios, dependiendo del contexto en el que se encuentre su organización, puedan resolver las necesidades específicas sin recurrir a la lectura completa de todo el documento.

A partir de lo que plantea el manual (IUMSS) en el capítulo 3, se realizó la integración de los requisitos de las normas NTC ISO 14001:2015, NTC ISO 45001:2018 y NTC ISO 13485:2016 teniendo en cuenta las cuatro etapas y once subetapas descritas en la tabla 2. Adicionalmente, se tuvo en cuenta que las últimas actualizaciones de las normas en gestión ambiental y de seguridad y salud en el trabajo presentan la estructura de alto nivel y que la norma NTC ISO 13485:2016 para dispositivos médicos no cuenta con ella, pero es homóloga al sistema de gestión de la calidad NTC ISO 9001:2015.

Finalmente, se realizó la validación de la metodología de integración de las normas a través del método del ábaco de Régnier, el cual tiene como objetivo interrogar a expertos en un tema específico y tratar sus respuestas en tiempo real o por vía remota a partir de una escala de colores. Como todos los métodos compuestos por expertos, estos se destinan a reducir la incertidumbre, confrontar un punto de vista grupal y, a la vez, tomar conciencia de la mayor o menor variedad de opiniones (Blogs, 2013). Para aplicar dicha metodología, se establecieron cuatro etapas, las cuales se describen en la tabla 1, a continuación: 
Tabla 1. Etapas Metodologías de Validación

\begin{tabular}{|c|c|c|}
\hline \multicolumn{3}{|c|}{ Herramienta de Validación } \\
\hline Etapa & Tema & Descripción \\
\hline 1 & Expertos técnicos sector comercialización de dispositivos médicos. & $\begin{array}{c}\text { Recolección de datos. } \\
\text { Nombres y apellidos. } \\
\text { Profesión. } \\
\text { Cargo o área de desempeño. }\end{array}$ \\
\hline 2 & Introducción. & $\begin{array}{l}\text { Representación de la problemática abordada. } \\
\text { Objetivos específicos a evaluar. } \\
\text { Descripción instructivo de diligenciamiento. }\end{array}$ \\
\hline 3 & Contextualización. & Descripción por fases del sistema de gestión integrado. \\
\hline 4 & Instrumento de validación propuesto. & $\begin{array}{c}\text { Valoración de la herramienta con selección múltiple de } \\
\text { colores según opción, para asignar el criterio que cada } \\
\text { experto de acuerdo a las afirmaciones planteadas. Se } \\
\text { realiza a nivel general una matriz de comparación } \\
\text { entre: } \\
\text { Listado de afirmaciones sobre la propuesta. } \\
\text { Listado de expertos. } \\
\text { Apreciaciones: espacio para sugerencias y comentarios } \\
\text { adicionales de los expertos. }\end{array}$ \\
\hline
\end{tabular}

Fuente: elaboración propia de los autores.

Signos, Investigación en Sistemas de Gestión

ISSN: 2145-1389 | e-ISSN: 2463-1140 | DOI: https://doi.org/10.15332/24631140

Vol. 13 N. ${ }^{\circ} 2$ | julio-diciembre de 2021 


\section{Resultados y discusión}

\section{Desarrollo de las etapas de integración propuestas en el manual IUMSS}

Como se mencionó anteriormente, el manual ha sugerido la integración como un proceso de cuatro etapas que se fraccionan a su vez en once subetapas a considerar si se pretende realizar una correcta metodología de integración:

Tabla 2. Etapas metodología de integración según el IUMSS

\begin{tabular}{|c|c|c|c|}
\hline N. ${ }^{\circ}$ & Clasificación etapa & N. ${ }^{\circ}$ & Sub-etapas \\
\hline \multirow{3}{*}{1} & \multirow{3}{*}{ Preparación. } & 1 & Liderar la integración. \\
\hline & & 2 & $\begin{array}{c}\text { Determinar el alcance de la } \\
\text { integración. }\end{array}$ \\
\hline & & 3 & Plan de integración. \\
\hline \multirow{3}{*}{2} & \multirow{3}{*}{ Conexión. } & 4 & $\begin{array}{c}\text { Estructurar el sistema de } \\
\text { gestión. }\end{array}$ \\
\hline & & 5 & $\begin{array}{c}\text { Estructurar el sistema de } \\
\text { gestión de requisitos } \\
\text { normativos. }\end{array}$ \\
\hline & & 6 & $\begin{array}{l}\text { Mapear el sistema de gestión } \\
\text { de requisitos normativos } \\
\text { contra el sistema de gestión. }\end{array}$ \\
\hline \multirow{3}{*}{3} & \multirow{3}{*}{ Incorporación. } & 7 & Identificar y analizar brechas. \\
\hline & & 8 & Cerrar brechas. \\
\hline & & 9 & Verificar cierre de brechas. \\
\hline \multirow[t]{2}{*}{4} & \multirow[t]{2}{*}{ Mantenimiento. } & 10 & $\begin{array}{l}\text { Mantener y mejorar la } \\
\text { integración. }\end{array}$ \\
\hline & & 11 & Aplicar lecciones aprendidas. \\
\hline
\end{tabular}

Fuente: elaboración propia de los autores a partir del IUMSS. 


\section{Etapa 1: preparación}

Subetapa 1: liderar la integración.

Toda metodología de integración debe basarse en un liderazgo organizacional. Este debe surgir como una decisión tomada por la dirección frente al contexto de una organización a partir de una necesidad estratégica, operativa o por el grado de madurez en la implementación de los diferentes sistemas de gestión. Liderar implica reconocer los beneficios y considerar las consecuencias durante las etapas de la integración.

En esta subetapa de liderazgo, es necesario proporcionar instrucciones para la integración de los requisitos de las normas en el sistema de gestión, por lo tanto, se requiere de lo descrito en la tabla 3, titulada instrucciones desde el liderazgo para la integración.

Tabla 3. Instrucciones desde el liderazgo para la integración

1. Identificar las necesidades de integración.

2. Adaptar la política de la organización para incluir la integración.

4. Establecer los objetivos para la integración.

\section{Determinar los riesgos y oportunidades de la integración.}

3. Determinar las iniciativas estratégicas para la integración.

6. Comunicar la decisión de integrar.

Fuente: elaboración propia de los autores a partir del IUMSS.

La decisión de liderazgo, basada en estas instrucciones, proporcionará una política integrada que direccionará la planificación, los recursos, las responsabilidades, el monitoreo y las comunicaciones durante la integración. Este enfoque debe ser sistémico y debe apoyarse desde la alta dirección, a través de la inclusión de elementos de la planificación estratégica como de la asignación de recursos, la promoción de una cultura organizacional y el diseño para la gestión conjunta de procesos clave (Asif et ál., 2009). 
Desde la estructura de alto nivel de la ISO, el liderazgo es una orientación al logro de los resultados previstos. Para la norma ISO 13485, orientada hacia la gestión de calidad para dispositivos médicos, esta estructura aún no se encuentra definida, aunque en el numeral 5.1 "compromiso de la dirección” se abarca el liderazgo que debe asumir la alta dirección desde su sistema de gestión de calidad, lo cual aportará al proceso de integración estrategias como la toma de decisiones, al considerar las necesidades de todas las partes interesadas.

Subetapa 2: determinar el alcance de la integración

Una vez la alta dirección ha tomado la decisión de integrar, deberá asumir el liderazgo y el compromiso de incorporar en una sola gestión varios esquemas normativos a su organización y, para ello, debe establecer un alcance en el proceso de integración, teniendo en cuenta los tiempos e impactos esperados. El alcance de cada organización dependerá del número de normas a integrar, el orden y los niveles de integración entre procesos, objetivos y recursos utilizados en el sistema de gestión.

El alcance de la integración se relaciona en gran medida con ciertos impulsores, que según el IUMMS son los que incentivan a la metodología de integración y, de acuerdo con ellos, se determina la necesidad de realizar dicho proceso, estos se describen a continuación:

- Requisitos del cliente: expectativa, necesidad u oportunidad de mercado.

- Necesidad interna: oportunidad de mejorar la eficiencia y eficacia de la organización.

- Regulaciones: cumplimiento de demandas, legislativas o administrativas de entes reguladores o agencias gubernamentales. La identificación de estos impulsores implica reconocer entonces los problemas externos y el entorno operativo del sector. 
Cualquier organización que tenga implementado o no un sistema de gestión podrá emplear el manual como una herramienta directriz para la metodología de integración de los diferentes esquemas normativos que desee incluir en su sistema.

Una vez establecido el alcance, se deberá planificar la integración teniendo en cuenta los riesgos y oportunidades relacionados. Para esto, se propone realizar un plan de integración que permitirá comenzar de manera más efectiva y eficiente el proceso de integración, como se muestra en la tabla 4, descrito a continuación:

Tabla 4. Plan de integración

\begin{tabular}{|c|c|}
\hline Variables & Características \\
\hline Propietario & $\begin{array}{c}\text { ¿Quién es el propietario del proyecto? Responsables de lanzar e } \\
\text { implementar el proyecto. }\end{array}$ \\
\hline Comité del proyecto & $\begin{array}{c}\text { Equipo multifuncional compuesto por personas que tienen la } \\
\text { habilidad, el conocimiento y la disponibilidad para completar el } \\
\text { trabajo. }\end{array}$ \\
\hline Líder del proyecto & Profesional experto en proyectos de mejora. \\
\hline Estrategia de comunicación & $\begin{array}{l}\text { Creada a partir de la conciencia organizativa y la coherencia de } \\
\text { la dirección estratégica a demás áreas de la organización. }\end{array}$ \\
\hline Riesgos y oportunidades & Relacionados con el proyecto de integración. \\
\hline Recursos & $\begin{array}{l}\text { Personal capacitado, instalaciones adecuadas, sistemas, } \\
\text { información, equipos de apoyo. }\end{array}$ \\
\hline Actividades de integración & $\begin{array}{c}\text { Descripción de los pasos detallados con roles y } \\
\text { responsabilidades asignados y cronograma para alcanzar los } \\
\text { objetivos del proyecto. }\end{array}$ \\
\hline
\end{tabular}

Fuente: elaboración propia de los autores a partir del IUMSS.

Aunque las actividades de integración se explicarán más a fondo en la etapa 2 (conexión), subetapas 4, 5 y 6, estas deberán definirse desde el plan de integración al hacer referencia a lo que describe el (IUMSS) en la página 57: 
- Definir el modelo del sistema de gestión que se adaptará al alcance de la integración.

- Configurar los requisitos de las normas para integrarse en el sistema de gestión.

- Mapear o vincular los requisitos de las normas con el sistema de gestión definido (procesos) de la organización.

Existen otras actividades que, de igual manera, se explicarán al detalle en la etapa 3 (incorporación), subetapas 7, 8 y 9, las cuales también deberán definirse desde el plan de integración y hacen referencia a:

- Analizar las brechas, incluida la identificación del nivel de conformidad e integración o la ausencia de procesos de la organización para cumplir con los requisitos.

- Cerrar las brechas.

- Verificar que el cierre de brechas funcione.

Finalmente, se plantean otras actividades que también se abordarán en la etapa 4 (mantenimiento), subetapas 10 y 11, las cuales también deberán definirse desde el plan de integración y hacen referencia a:

- $\quad$ Medir, monitorear y mejorar continuamente al determinar los indicadores clave del proceso, y revisar el desempeño y el progreso

- $\quad$ Centrarse en el aprendizaje al identificar y aprovechar las oportunidades para una integración más efectiva y eficiente

\section{Etapa 2: conexión}

Subetapa 4: estructurar el sistema de gestión

Se hace necesaria la creación de la estructura de un sistema de gestión que funcionará como soporte y, a su vez, facilitará la comprensión de cualquier escenario. 
Subetapa 5: estructurar el sistema de gestión de requisitos normativos

El primer paso para vincular los requisitos de las normas con el sistema de gestión en una organización es considerar las relaciones entre los diferentes procesos, recursos y objetivos. Esto implica comprender la conexión de los procesos que se realizan durante la fabricación de productos o la prestación de un servicio con los demás procesos de soporte y, de la misma manera, con las necesidades de las partes interesadas. Por esta razón, es conocido que las organizaciones gestionen sus actividades, recursos y objetivos a través del enfoque de la gestión por procesos.

Para cumplir con esta subetapa de la metodología de integración, se realizó una comparación entre las tres normas a integrar a través de una matriz que se muestra parcialmente en la tabla 5. En ella se tomaron todos los requisitos aplicables de cada norma y se identificaron los puntos en común (los requisitos similares que permiten la integración), los homólogos (los requisitos que tienen puntos similares solo en dos de los tres esquemas normativos) y específicos (los requisitos relacionados directamente a una norma en particular). Estos últimos deben ser armonizados dentro del sistema integrado de gestión (ver tabla 5). 
Tabla 5. Matriz de comparación de requisitos

\begin{tabular}{|c|c|c|c|c|c|}
\hline & \multirow{2}{*}{ Requisitos aplicables } & \multicolumn{3}{|c|}{ Sistemas de gestión a integrar numerales de las normas } & \multirow{2}{*}{$\begin{array}{l}\text { Consideración del } \\
\text { requisito }\end{array}$} \\
\hline & & $\begin{array}{l}\text { NTC ISO 13485: } \\
2016\end{array}$ & $\begin{array}{c}\text { NTC ISO } \\
14001: 2015\end{array}$ & $\begin{array}{c}\text { NTC ISO } \\
45001: 2018\end{array}$ & \\
\hline \multirow{4}{*}{ 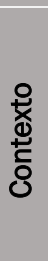 } & Comprensión del sector y su contexto. & 4 & 4.1 & 4.1 & Común. \\
\hline & $\begin{array}{l}\text { Comprensión de los requisitos y de las partes } \\
\text { interesadas. }\end{array}$ & 4 & 4.2 & 4.2 & Común. \\
\hline & Alcance del sistema integrado de gestión. & 4 & 4.3 & 4.3 & Común. \\
\hline & El sistema integrado de gestión. & 4.1 & 4.4 & 4.4 & Homólogo. \\
\hline \multirow{5}{*}{ 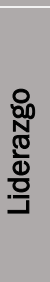 } & Liderazgo y compromiso. & 5.1 & 5.1 & 5.1 & Común. \\
\hline & Enfoque al cliente. & 5.2 & * & * & Específico. \\
\hline & Política integrada. & 5.3 & 5.2 & 5.2 & Común. \\
\hline & Roles responsabilidades y autoridades. & 5.5 .1 y 5.5 .2 & 5.3 & 5.3 & Común. \\
\hline & Consulta participativa de los trabajadores. & * & * & 5.4 & Específico. \\
\hline \multirow{6}{*}{ 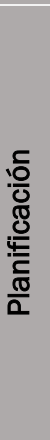 } & Riesgos y oportunidades. & 5.4 .2 & 6.1 & 6.1 & Homólogo. \\
\hline & $\begin{array}{l}\text { Identificación y evaluación de aspectos } \\
\text { ambientales. }\end{array}$ & * & 6.1 .2 & * & Específico. \\
\hline & $\begin{array}{l}\text { Identificación de peligros, evaluación de riesgos } \\
\text { laborales y planificación de acciones. }\end{array}$ & * & * & 6.1 .2 & Específico. \\
\hline & Requisitos legales y otros requisitos. & $\begin{array}{l}4,5.2,7.2 .2,7.3 .3 \\
\quad 7.4 .1 \text { y } 8.2 .3\end{array}$ & 6.1 .3 y 9.1 .2 & 6.1 .3 y 9.1 .2 & Homólogo. \\
\hline & Objetivos del programa. & 5.4 .1 & 6.2 & 6.2 & Común. \\
\hline & Planificación de los cambios. & 5.4 .2 y 7.3 .9 & $6.1 .2,7.4 .2$ y 8.1 & 8.1 .3 & Homólogo. \\
\hline
\end{tabular}

Fuente: elaboración propia.

Signos, Investigación en Sistemas de Gestión

ISSN: 2145-1389 | e-ISSN: 2463-1140 | DOI: https://doi.org/10.15332/24631140

Vol. 13 N.o 2 | julio-diciembre de 2021 
Por otra parte, es importante considerar que cuando existe un sistema de gestión ya implementado dentro de una organización y se desea integrar un nuevo sistema, es necesario comparar primero los requisitos del sistema a integrar con los que ya se encuentran establecidos y del mismo modo se deberán analizar sus requisitos. Esto proporciona un enfoque eficaz y eficiente para la integración de nuevos esquemas normativos (ISO, 2018).

Subetapa 6: mapear el sistema de gestión de requisitos normativos contra el sistema de gestión

Según el manual (ISO, 2018), mapear es la manera como se genera valor, porque se enfrentan los requisitos de las normas con los procesos, recursos y objetivos que se tienen estipulados en el sistema de gestión. De esta manera resulta una tarea útil para minimizar las redundancias y maximizar la sinergia de la organización.

Para dar cumplimiento a esta subetapa del proceso de integración, se tuvieron en cuenta los requisitos comunes, homólogos y específicos reagrupados en una nueva matriz (ver tabla 6), la cual se muestra parcialmente. En la columna vertical de la izquierda se ubicaron los procesos que hipotéticamente podrían tener una organización y los subprocesos más relevantes identificados también de manera genérica (Calso Morales y Álvarez, 2018) para ver las consideraciones del requisito (ver tabla 7). En la fila horizontal en la parte superior se representan las cláusulas que contienen los requisitos de las normas y se detallan los numerales correspondientes a cada norma. Seguido a eso, se analizó el impacto de cada uno de los requisitos sobre los procesos con las preguntas sugeridas por el IUMMS dadas a continuación: 
- ¿Dónde se encuentran oportunidades de integración al revisar los procesos, recursos y objetivos de la organización con los requisitos de las normas?

- ¿Cómo se cumplen estos requisitos en los procesos al revisar las normas que tienen puntos en común?

- Teniendo en cuenta la pregunta anterior, ¿qué procesos se verían afectados al revisar los requisitos específicos de una norma?

- ¿Qué proceso se vería afectado al revisar los requisitos específicos de una norma?

- Teniendo en cuenta la pregunta anterior, ¿̇se necesitarán nuevos procesos? y, de ser así, ¿cómo puede la organización integrar mejor estos requisitos en su sistema de gestión?

Finalmente, se señalaron con un símbolo de aprobado todos aquellos requisitos que tienen impacto sobre los procesos y se indica su unificación con el sistema de gestión. Adicionalmente, esta subetapa permitió:

- Detectar procesos que no se relacionan con los requisitos de las normas.

- Identificar redundancias en los procesos.

- Determinar los procesos mínimos necesarios que satisfagan los requisitos normativos.

- $\quad$ Establecer el nivel de comunidad en los requisitos de las normas. 
Tabla 6. Matriz de mapeo de requisitos

\begin{tabular}{|c|c|c|c|c|c|}
\hline \multicolumn{2}{|c|}{ Procesos } & \multicolumn{2}{|c|}{ Contexto } & \multicolumn{2}{|c|}{ Liderazgo } \\
\hline & Procesos & $\begin{array}{l}\text { Compresión del sector } \\
\text { y su contexto }\end{array}$ & $\begin{array}{l}\text { Compresión de los } \\
\text { requisitos y de las } \\
\text { partes interesadas }\end{array}$ & $\begin{array}{l}\text { Liderazgo y } \\
\text { compromiso }\end{array}$ & Enfoque al cliente \\
\hline \multirow{2}{*}{ Requisitos } & \multirow[b]{3}{*}{$\begin{array}{l}\text { Logística y } \\
\text { apoyo }\end{array}$} & $\begin{array}{c}\text { NTC ISO } \\
13485 / 14001 / 45001\end{array}$ & $\begin{array}{c}\text { NTC ISO } \\
13485 / 14001 / 45001\end{array}$ & $\begin{array}{c}\text { NTC ISO } \\
13485 / 14001 / 45001\end{array}$ & NTC ISO 13485 \\
\hline & & 4/4.1/4.1 & $4 / 4.2 / 4.2$ & 5.1 & 5.2 \\
\hline Gestión de operaciones & & $\square$ & $\square$ & & $\square$ \\
\hline \multirow{6}{*}{$\begin{array}{l}\text { Calidad y gestión } \\
\text { integrada }\end{array}$} & $\begin{array}{l}\text { Sistema } \\
\text { integrado de } \\
\text { gestión }\end{array}$ & $\square$ & $\square$ & $\square$ & $\square$ \\
\hline & $\begin{array}{l}\text { Gestión } \\
\text { ambiental }\end{array}$ & $\square$ & $\square$ & $\square$ & \\
\hline & $\begin{array}{l}\text { Gestión } \\
\text { seguridad y } \\
\text { salud en el } \\
\text { trabajo }\end{array}$ & $\square$ & $\square$ & $\square$ & \\
\hline & $\begin{array}{l}\text { Gestión de } \\
\text { calidad } \\
\text { dispositivos } \\
\text { Médicos }\end{array}$ & $\square$ & $\square$ & $\square$ & \\
\hline & $\begin{array}{c}\text { Aseguramiento } \\
\text { de calidad }\end{array}$ & & & & \\
\hline & $\begin{array}{l}\text { Gestión de } \\
\text { asuntos } \\
\text { regulatorios }\end{array}$ & $\square$ & $\square$ & & $\square$ \\
\hline
\end{tabular}

Fuente: elaboración propia de los autores.

Signos, Investigación en Sistemas de Gestión

ISSN: 2145-1389 | e-ISSN: 2463-1140 | DOI: https://doi.org/10.15332/24631140

Vol. 13 N. ${ }^{\circ} 2$ | julio-diciembre de 2021 
Tabla 7. Requisitos homólogos, comunes y específicos

\begin{tabular}{|c|c|}
\hline \multicolumn{2}{|r|}{ Consideraciones del requisito } \\
\hline Común & $\begin{array}{l}\text { Requerimientos similares que permiten la integración teniendo en cuenta la } \\
\text { aplicación de los tres sistemas de gestión. }\end{array}$ \\
\hline Específico & $\begin{array}{l}\text { Requisitos relacionados directamente con las exigencias de la norma en particular y } \\
\text { que deben ser armonizados con el sistema de gestión integrado. }\end{array}$ \\
\hline Homólogo & $\begin{array}{c}\text { Requerimientos que tienen puntos similares solo en dos de los tres sistemas de } \\
\text { gestión integrado. }\end{array}$ \\
\hline * & No Aplica \\
\hline
\end{tabular}

Fuente: Elaboración propia de los autores.

\section{Etapa 3: incorporación}

Subetapa 7: identificar y analizar brechas

El objetivo de esta subetapa es identificar y comprender el alcance de las diferencias entre el sistema de gestión existente y los requisitos establecidos en las normas, al verificar si los procesos que se han definido en la organización cumplen y en qué medida con los requisitos, siempre y cuando exista la intención de certificarse o solamente mejorar el desempeño y la sostenibilidad. También es importante, porque evalúa el nivel de integración logrado una vez que los requisitos se han incorporado a la organización. Tales brechas pueden eliminarse posteriormente con una política, proceso, procedimiento o práctica apropiada. Después de este análisis, será posible minimizar aún más las redundancias, las actividades y recursos innecesarios.

Para dar cumplimiento a esta subetapa del proceso de integración fue necesario analizar y comprender los procesos que puede tener una organización, teniendo en cuenta el nivel de cumplimiento de los requisitos de las normas integradas. Esto debido a que cada sistema de gestión presentará variaciones en la información almacenada por la 
naturaleza de cada norma. Es importante señalar que el análisis de brechas no es una tarea fácil, ya que requiere de la habilidad y colaboración de toda una organización para llegar a acuerdos definidos y poder armonizar el flujo de información para cada proceso según los requisitos de las normas. Sin embargo, aunque el proceso de integración no haya sido aplicado en una organización y se requieran procesos bien documentados para esta labor, este análisis se realizará de acuerdo con lo descrito en el IUMSS teniendo en cuenta el enfoque de sistemas integrados (centrarse en los procesos de la organización) y que sigue los pasos que son relevantes para una auditoría y autoevaluaciones típicas de un sistema de gestión:

- Identificar y comprender los requisitos de las normas del sistema de gestión.

- Recopilar y verificar información sobre el sistema de gestión.

- Comparar la información del sistema de gestión con los requisitos y evidencias de cumplimiento.

- Identificar las oportunidades de integración, superposiciones y sinergias a través de:

- Los requisitos de diferentes normas.

- Los diferentes componentes del sistema de gestión (por ejemplo, procesos, recursos y objetivos).

Como se puede observar, el análisis de brechas es un informe detallado de las subetapas 5 y 6 que puede incluir:

- El propósito del análisis de brechas.

- El enfoque del análisis de las brechas.

- Una matriz de análisis de brechas.

- Un listado de los requisitos normativos del sistema de gestión analizado. 
- Un listado de los procesos analizados.

- Información documentada revisada.

- Personas entrevistadas.

- Un resumen de los hallazgos.

- Acciones necesarias para cerrar las brechas.

- Cronograma y recursos para el cierre de brechas.

- Recomendaciones.

En la misma matriz donde se realizó el mapeo de requisitos (ver tabla 8) se podrán identificar con una escala de colores los procesos que, luego del análisis realizado, presenten un cumplimiento total, parcial, de no cumplimiento o de no aplicar, como se muestra a manera de ejemplo en la matriz de mapeo de la subetapa 6.

Tabla 8. Matriz de identificación y análisis de brechas

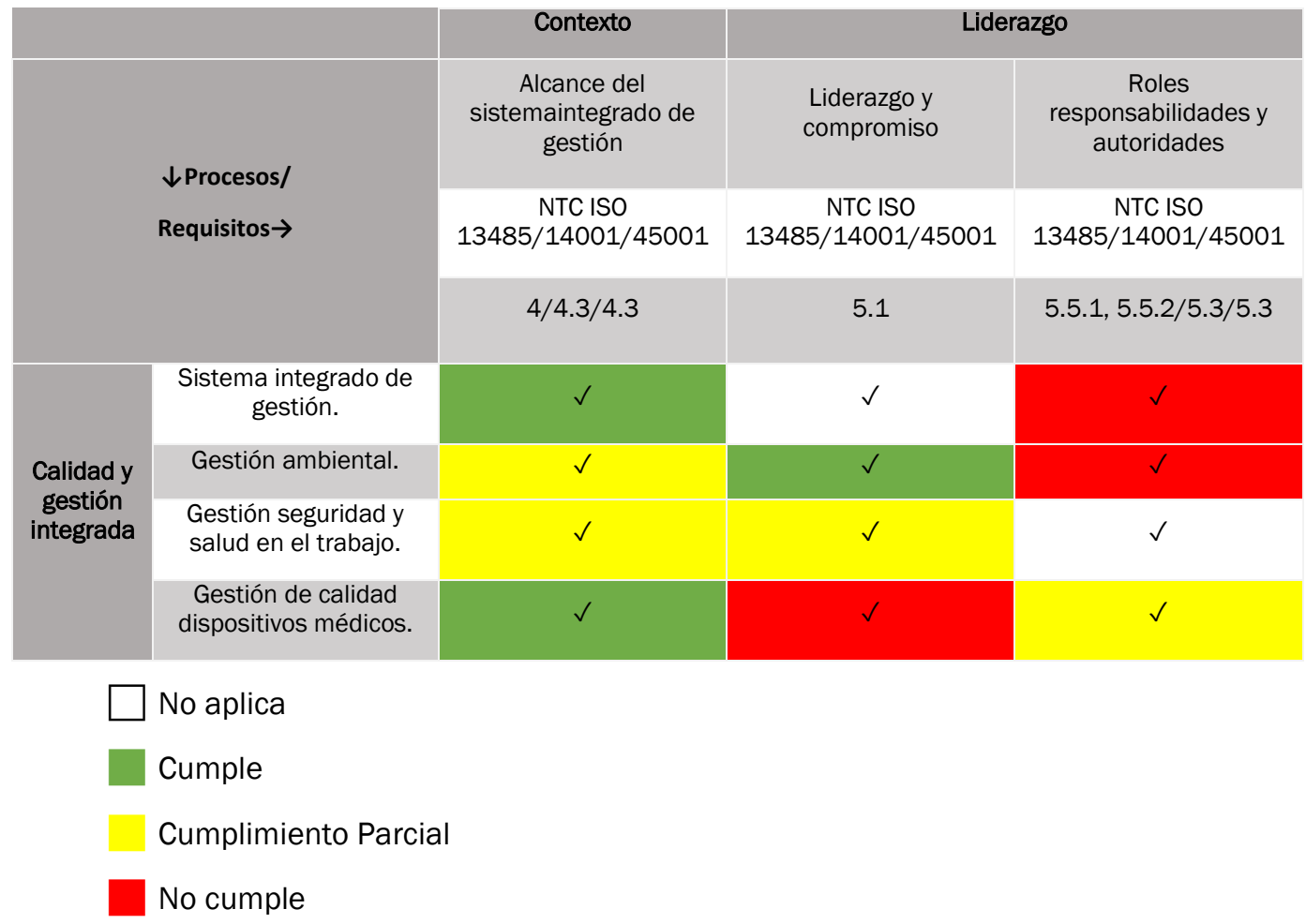

Fuente: elaboración propia de los autores.

Signos, Investigación en Sistemas de Gestión

ISSN: 2145-1389 | e-ISSN: 2463-1140 | DOI: https://doi.org/10.15332/24631140

Vol. 13 N.० 2 | julio-diciembre de 2021 
Una vez realizado el informe que describe detalladamente las brechas existentes, es necesario establecer un plan para su cierre, el cual deberá incluir objetivos e indicadores.

Las brechas encontradas serán consideras como fallas en la comprensión al momento de aplicar un requisito normativo y, para ello, deberán identificarse qué tipo de debilidades sistémicas se están relacionando con problemas organizacionales más amplios, como las interrelaciones entre los procesos insuficientemente definidas o ausencia de procesos que aborden los requisitos de las normas; así mismo, también pueden existir oportunidades de mejora relacionadas con el uso integrado de las normas. Los pasos a seguir para el cierre de brechas pueden incluir:

- Determinar las acciones correctivas y de mejora con base a los resultados del análisis de brechas.

- Identificar los componentes del sistema de gestión afectado y determinar las acciones correctivas necesarias para cerrar las brechas como: la información documentada, la necesidad de capacitación, etc.

- $\quad$ Realizar acciones de mejora: esto debe incluir abordar las oportunidades de integración de múltiples requisitos o componentes normativos del sistema de gestión en uno solo, por ejemplo, al combinar varios procesos para el control de la información documentada o unificar políticas específicas de funciones.

Teniendo en cuenta lo anterior, a continuación, se describen los tipos de brechas que pueden llegar a existir (ver figura 1). 
Figura 1. Tipos de brechas

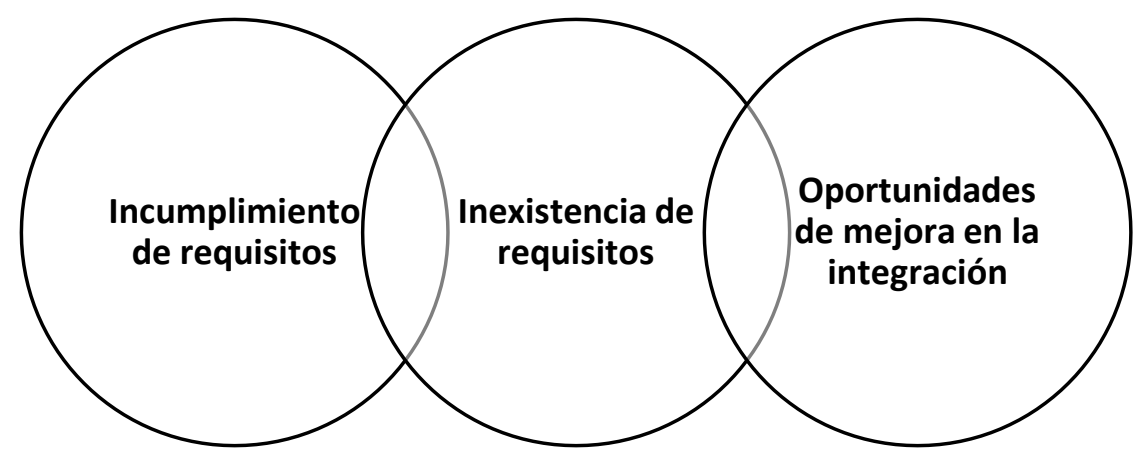

Fuente: elaboración propia.

Como ya se había mencionado, realizar el ejercicio de identificar y analizar brechas en el presente trabajo no pudo llevarse a cabo, debido a que no se cuenta con la información proveniente de una organización; sin embargo, se plantearán algunos pasos importantes que en la práctica ayudarán a realizar el cierre de las brechas encontradas en la tabla 9, a continuación:

Tabla 9. Consideraciones cierre de brechas

\begin{tabular}{|c|c|c|}
\hline N. ${ }^{\circ}$ & Acciones & Descripción \\
\hline 1. & $\begin{array}{l}\text { Discutir los resultados del análisis } \\
\text { de las brechas con los empleados. }\end{array}$ & $\begin{array}{c}\text { Indagar con los jefes de proceso y demás colaboradores } \\
\text { si están de acuerdo con el tipo de hallazgo y que } \\
\text { medidas tomarían al respecto. }\end{array}$ \\
\hline 2. & $\begin{array}{l}\text { Priorizar los resultados según la } \\
\text { relevancia para el negocio. }\end{array}$ & $\begin{array}{l}\text { Determinar el número de brechas existentes. } \\
\text { ¿A cuántos procesos y objetivos afectan? } \\
\text { ¿Qué recursos se requieren para cerrarlas? } \\
\text { ¿Qué procesos haría falta establecer para dar } \\
\text { cumplimiento a los requisitos? }\end{array}$ \\
\hline 3. & $\begin{array}{c}\text { Determinar las acciones necesarias } \\
\text { y responsables }\end{array}$ & $\begin{array}{l}\text { Cada brecha deberá tener un propietario en el proceso } \\
\text { donde se presentó la falencia con el requisito aplicable. }\end{array}$ \\
\hline 4. & $\begin{array}{l}\text { Implementar y documentar las } \\
\text { acciones tomadas }\end{array}$ & $\begin{array}{l}\text { Haciendo uso de las matrices existentes para llevar un } \\
\text { control y seguimiento de cada acción. }\end{array}$ \\
\hline
\end{tabular}

Signos, Investigación en Sistemas de Gestión

ISSN: 2145-1389 | e-ISSN: 2463-1140 | DOI: https://doi.org/10.15332/24631140

Vol. 13 N.० 2 | julio-diciembre de 2021 


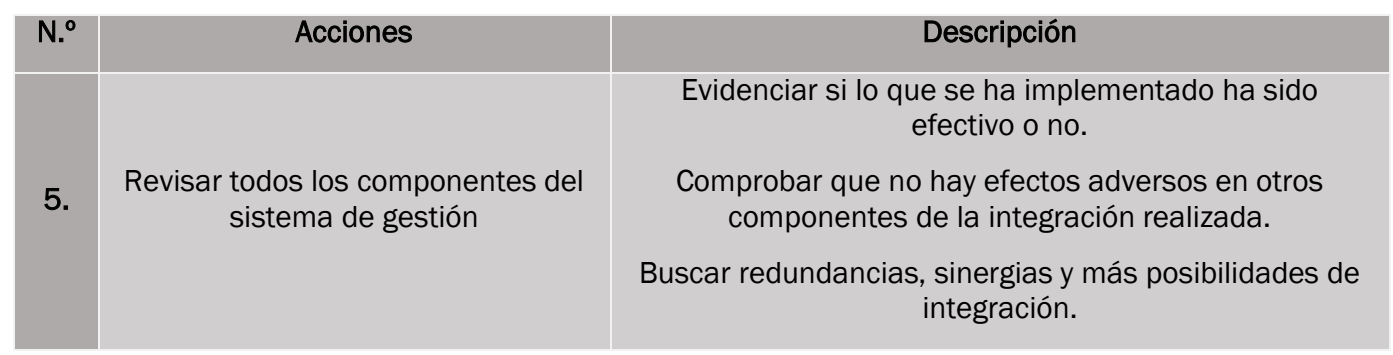

Fuente: elaboración propia de los autores a partir de la IUMSS.

Subetapa 9: verificar el cierre de brechas.

Según el (ISO, 2018), el desempeño del sistema de gestión de la organización a lo largo del tiempo es el verdadero indicador de que el sistema de gestión funcione correctamente. Existen medidas internas dentro de la organización que pueden ser indicadores principales del desempeño organizacional frente a los objetivos. Las organizaciones necesitan observar los procesos, los recursos y los objetivos teniendo en cuenta los vínculos entre las funciones y los requisitos de las normas del sistema de gestión integrado.

Para confirmar el cierre de cualquier brecha y mantener las mejoras realizadas, las organizaciones deben revisar los resultados a través de auditorías internas y la revisión de la gestión. Una vez que se cierra la brecha, es importante verificar si las acciones de implementación continúan y poder determinar si los beneficios se han alcanzado.

Las evaluaciones de los requisitos normativos del sistema de gestión proporcionan información importante y direccionan a las organizaciones a conocer su nivel de desempeño y determinar si las acciones correctivas han sido efectivas. Una vez que se han implementado dichas acciones, se podrá llevar entonces una auditoría interna o una autoevaluación centrada en las brechas identificadas y las acciones correctivas relacionadas, para así garantizar una implementación eficaz y eficiente. 
Subetapa 10: mantener y mejorar la integración

Después que una organización completa el análisis de brechas y cierra con éxito las brechas identificadas, el siguiente paso es garantizar que los requisitos del sistema de gestión permanezcan implementados adecuadamente. El valor de la integración se refleja en el desempeño de la organización. Verificar la implementación mediante el monitoreo y la revisión periódica del sistema es una parte integral del mantenimiento y la mejora de un sistema de gestión eficaz (IUMSS). Para ello se recomienda tener en cuenta lo siguiente:

- Verificar el compromiso continuo de la alta dirección.

- Revisar y actualizar la documentación.

- Revisión de los riesgos y las oportunidades encontradas.

- Buscar áreas donde se pueda agregar valor.

- Buscar mejores formas de usar los recursos.

- Verificar que todo esté lo más integrado posible.

- Verificar el cumplimiento de los objetivos.

- Considerar siempre la mejora continua.

- Tratar de mantener beneficios de manera sostenible.

- Monitorear los requisitos de las partes interesadas.

- Validar los procesos.

- Verificar cambios en los requisitos legales.

Subetapa 11: aplicar lecciones aprendidas

A medida que las organizaciones pasan por el proceso de integración, hay muchas lecciones que aprender y nuevos desafíos que pueden surgir. No existe una única forma de lograr la integración o mantener y mejorar un sistema de gestión integrado. Las organizaciones deben seguir mejorando mediante la comprensión de los resultados que se han obtenido. 
Estas lecciones y desafíos pueden usarse para mejorar las metodologías de integración actuales o futuros. Además, se pueden aprovechar como una oportunidad para mejorar otros aspectos como:

- Gestión del conocimiento.

- Resistencia al cambio.

- Competencias.

- Diferencias en las competencias profesionales.

- Relacionar mejor los requisitos con las exigencias de las partes interesadas.

Al comparar la metodología de la IUMSS, con elementos de otras propuestas de integración de sistemas de gestión, se puede evidenciar una relación cercana entre la estructura propia del manual propuesto por la ISO y los principios rectores determinados por Nunhes et ál., (2019). Ellos analizaron 28 elementos en común identificados a lo largo de la literatura más referenciada entre el 2006 y 2016 y encontraron que:

- Gestión sistémica.

- Estandarización.

- Integración estratégica, táctica y operativa.

- Aprendizaje organizacional.

- Desburocratización.

- Mejora continua.

Resultan ser los pilares en el desarrollo y mantenimiento de los sistemas integrados de gestión, los cuales se encuentran contenidos implícitamente en cada una de las etapas dadas en la metodología de integración que propone el manual IUMSS. 


\section{Desarrollo de la metodología de validación}

Tal como se planteó en la tabla 1, la metodología de validación se realizó a través de cuatro etapas que permitieron obtener las siguientes apreciaciones:

\section{Etapa 1: expertos técnicos}

Para esta etapa participaron un total de siete expertos con perfiles y experiencia seleccionadas en relación con la gerencia de calidad o dirección técnica en organizaciones pequeñas, medianas y a nivel multinacional que se dedican a la comercialización de dispositivos médicos en Colombia.

\section{Etapa 2: introducción}

Se presentó la metodología de integración de manera introductoria a cada uno de los expertos, describiendo la problemática general y explicando por qué se desarrollaron las etapas de integración de las normas NTC ISO 13485:2016, NTC ISO 14001:2015 y NTC ISO 45001:2018.

Como se mencionó anteriormente, la herramienta implementada fue el ábaco de Régnier, que consta de una gama de colores alineados según la convención internacional adoptada para los colores del semáforo. Estos colores indican la opinión de cada experto respecto a las afirmaciones realizadas con base en los modelos propuestos. A nivel general, los colores van desde el verde hasta el negro, desde las opiniones positivas, pasando a las posiciones neutras, luego opiniones negativas y, por último, condicionales como el voto en blanco y renuencia a participar. Así, tienen el siguiente significado descrito en la tabla 10: 
Tabla 10. Convención de colores y su significancia

\begin{tabular}{|c|c|}
\hline Clasificación de voto & Interpretación \\
\hline Muy favorable. & Opinión totalmente de acuerdo. \\
\hline Favorable. & Opinión de acuerdo. \\
\hline Neutro. & Opinión aceptable. \\
\hline Desfavorable. & Opinión en desacuerdo. \\
\hline Muy desfavorable. & Opinión totalmente en desacuerdo. \\
\hline Voto en blanco. & Prefiere no opinar. \\
\hline Renuencia a participar. & Impedido a opinar. \\
\hline
\end{tabular}

Fuente: elaboración propia de los autores.

Después se explicó la descripción del instructivo paso a paso, haciendo énfasis en los criterios de evaluación de acuerdo con la matriz y convención de colores, bajo la cual se selecciona la opción más ajustable a la opinión de cada experto (Martínez Narváez, 2018).

\section{Etapa 3: contextualización}

Se hizo una descripción de las fases de desarrollo. Todo con el fin de dar una contextualización general de la metodología de integración.

\section{Etapa 4: instrumento de validación propuesto}

Para la etapa cuatro, se solicitó a cada experto valorar cada una de las cinco afirmaciones planteadas, teniendo en cuenta la pertinencia de cada uno de los componentes de las propuestas realizadas para el entregable. 
Dichas afirmaciones y las evaluaciones respectivas de cada experto se pueden observar en la tabla 11.

Signos, Investigación en Sistemas de Gestión

ISSN: 2145-1389 | e-ISSN: 2463-1140 | DOI: https://doi.org/10.15332/24631140

Vol. 13 N.o 2 | julio-diciembre de 2021 
Tabla 11. Afirmaciones propuestas de integración

\begin{tabular}{|c|c|c|c|c|c|c|c|c|}
\hline \multicolumn{2}{|c|}{$\begin{array}{c}\text { Instrumento de validación basado en el método del } \\
\text { ábaco de Regnier }\end{array}$} & \multicolumn{7}{|c|}{ Expertos seleccionados } \\
\hline Ítem & $\begin{array}{c}\text { Afirmaciones sobre la metodología de } \\
\text { integración }\end{array}$ & a. & b. & c. & d. & e. & f. & g. \\
\hline 1. & $\begin{array}{l}\text { La propuesta del sistema integrado de } \\
\text { gestión que se propone a partir de la guía } \\
\text { IUMSS para el sector de dispositivos } \\
\text { médicos es una herramienta que ayudaría } \\
\text { al logro de los objetivos estratégicos de las } \\
\text { organizaciones comercializadoras en } \\
\text { Colombia. }\end{array}$ & Favorable & Favorable & Favorable & Favorable & Favorable & Favorable & $\begin{array}{c}\text { Muy } \\
\text { favorable }\end{array}$ \\
\hline 2. & $\begin{array}{l}\text { La propuesta de integración de los tres } \\
\text { sistemas de gestión: calidad, ambiental y } \\
\text { seguridad y salud en el trabajo para el } \\
\text { sector de comercialización de dispositivos } \\
\text { médicos evita el desgaste en tiempos, } \\
\text { duplicidad de procesos, incumplimiento de } \\
\text { requisitos y optimiza el uso eficiente de } \\
\text { recursos. }\end{array}$ & Favorable & Favorable & Favorable & Favorable & Favorable & $\begin{array}{c}\text { Muy } \\
\text { Favorable }\end{array}$ & $\begin{array}{c}\text { Muy } \\
\text { favorable }\end{array}$ \\
\hline 3. & $\begin{array}{c}\text { La metodología de ilntegración propuesta } \\
\text { por el IUMMS facilita la integración de los } \\
\text { procesos de la organización con los } \\
\text { estándares de las diferentes normas a } \\
\text { integrar. }\end{array}$ & $\begin{array}{c}\text { Muy } \\
\text { favorable }\end{array}$ & $\begin{array}{c}\text { Muy } \\
\text { favorable }\end{array}$ & Favorable & Favorable & $\begin{array}{c}\text { Muy } \\
\text { favorable }\end{array}$ & Favorable & $\begin{array}{c}\text { Muy } \\
\text { Favorable }\end{array}$ \\
\hline 4. & $\begin{array}{c}\text { El proceso de calidad y sistema integrado } \\
\text { propuesto en la estructura genérica del } \\
\text { sistema integrado de gestión optimizara } \\
\text { los procesos y los recursos de las } \\
\text { gestiones unificadas, reducir de la } \\
\text { burocracia y por consiguiente la reducir los } \\
\text { costos. }\end{array}$ & $\begin{array}{c}\text { Muy } \\
\text { favorable }\end{array}$ & $\begin{array}{c}\text { Muy } \\
\text { favorable }\end{array}$ & Favorable & Favorable & Favorable & $\begin{array}{c}\text { Muy } \\
\text { Favorable }\end{array}$ & $\begin{array}{c}\text { Muy } \\
\text { favorable }\end{array}$ \\
\hline
\end{tabular}

Signos, Investigación en Sistemas de Gestión

ISSN: 2145-1389 | e-ISSN: 2463-1140 | DOI: https://doi.org/10.15332/24631140

Vol. 13 N.0 2 | julio-diciembre de 2021 
Instrumento de validación basado en el método del ábaco de Regnier

El manual IUMSS permitirá realizar la

integración de otras nuevas normas

alineadas con la estructura de alto nivel de

la ISO o aquellas que permitan realizar su

equivalencia.

Fuente: elaboración propia.

Signos, Investigación en Sistemas de Gestión

ISSN: 2145-1389 | e-ISSN: 2463-1140 | DOI: https://doi.org/10.15332/24631140

Vol. 13 N.0 2 | julio-diciembre de 2021
Expertos seleccionados

$$
\text { Muy }
$$

favorable

Favorable

Muy 
A nivel general se puede observar en la tabla 11, que la herramienta planteada de validación fue evaluada obteniendo votos en promedio con concepto favorable, lo cual evidencia la conformidad de los expertos técnicos con la propuesta de integración bajo la metodología propuesta por el IUMSS.

Para los ítems 1 y 2, que tuvieron como resultado la calificación por expertos como favorable, y muy favorable, se afirma que la herramienta de integración IUMSS, implementada para la metodología de integración propuesta, permitirá alcanzar el logro de los objetivos estratégicos, evitará el gasto en tiempos de duplicidad de los procesos y el incumplimiento de requisitos, optimizando el uso eficiente de los recursos.

En cuanto a los ítems 3, 4 y 5 se obtuvieron resultados muy favorables y favorables, en los cuales se afirma que la metodología de integración propuesta por la IUMMS facilita la integración de los procesos de la organización con los estándares de las diferentes normas a integrar.

\section{Conclusiones}

Se evidencia, a través de la investigación realizada, una necesidad de interpretar, establecer y difundir herramientas metodológicas que faciliten el proceso de integración de diferentes sistemas de gestión, que sean flexibles a los requerimientos normativos y de una manera genérica sea aplicable a todo tipo de organización.

Se observa, a partir de los resultados obtenidos, que cualquier usuario podrá abordar el uso del manual IUMSS de manera diferente según el core organizacional y las metas previstas de los procesos; sin embargo, para que dicho abordaje sea exitoso deberá seguir una estructura lógica, la cual se plantea en este artículo. 
La centralidad en los procesos o el enfoque basado en procesos resulta fundamental en la integración de diferentes sistemas de gestión, debido a que se pone de manifiesto sobre qué tan alineados se encuentran los proceso con los requisitos de las normas, además de ser relevantes en las auditorías.

Se evidencia que, aunque la NTC ISO 13485:2016 no cuenta con una estructura de alto nivel definida, como en la NTC ISO 14001:2015 y NTC ISO 45001:2018, se pudo realizar la integración sin complicaciones, puesto que la norma relacionada a dispositivos médicos se encuentra asociada homólogamente a la norma NTC ISO 9001:2015.

La integración tiene un fin último, fundamentado en encontrar las relaciones existentes entre los requisitos normativos, procesos, recursos y objetivos con el propósito de identificarlos, evaluarlos y mejorarlos para aumentar el desempeño organizacional y disminuir la magnitud global del sistema.

El manual IUMSS resulta ser una herramienta pedagógica que podría utilizarse ampliamente en la enseñanza de sistemas de gestión, debido al lenguaje claro y estructurado que facilitaría a los docentes el entendimiento de conceptos claves en sus estudiantes.

Se logró traducir e interpretar el manual IUMSS para su aplicación en cualquier sector económico, teniendo en cuenta que Colombia no es un país bilingüe y que dicho manual solo está a la venta en el idioma inglés.

Los expertos técnicos consideran que la metodología dada por el IUMSS es una herramienta robusta y fácil de entender para la metodología de integración. 


\section{Referencias}

Asif, M., Brujin, E., Fisscher, O., Searcy, C., y Steenhuis, H. (2009). Process embedded design of integrated management systems. International Journal of Quality \& Reliability Management, 6(3), 261-282. https://www.emerald.com/insight/content/doi/10.1108/02656710910936735/full $\lfloor$ html

Bernardo, M., Gianni, M., Gotzamani, K., y Simón, A. (2017). Is there common pattern to integrate multiple management sistems? A comparative analysis between organizations in Greece and Spain. Journal of Cleaner Production, 151, 121-151. https://doi.org/10.1016/j.jclepro.2017.03.036

Bernardo, M., Simón , A., Tari, J. y Molina, A. (2015). Benefit of management sistems integration: a literatura review. Journal of Cleaner Production, 94, 260-267. https://doi.org/10.1016/j.jclepro.2015.01.075

Rochel Ojeda, J. A.. (2013, 29 de octubre). El ábaco de Reigner. Prospectiva Blogs. http://jrprospectiva.blogspot.com/2013/10/el-abaco-de-regnier.html

Cabrera, H., Medina León, A., Puente, J., Nogueira Rivera, D., y Nuñez Chaviano, Q. (2015). La integración de Sistemas de Gestión Empresariales, conceptos, enfoques y tendencias. Ciencias de la Información, 46(3), 3-8. https://biblat.unam.mx/hevila/Cienciasdelainformacion/2015/vol46/no3/1.pdf

Calso Morales, N., yPardo Álvarez, J. (2018). Guía práctica para la integración de Sistemas de Gestión. ISO 9001, ISO 14001 e ISO 45001. Aenor.

Gianni, A., Gotzamani, K., y Vouzas, K. (2017). Food integrated management systems: dairy industry insights. International Journal of Quality \& Reliability Management, 34(2), 194-215.

https://www.emerald.com/insight/content/doi/10.1108/IJQRM-05-2015oo76/full/html

Heras, I., Bernardo, M. y Casadesús, M. (2007). La integración de sistemas de gestión basados en estándares internacionales: resultados de un estudio empírico realizado en la capv1. Revista de Dirección y Administración de Empresas, 14, 155-174. https://www.ehu.eus/documents/2069587/2113837/14 11.pdf

ISO. (2018). The Integrated Use of Management System Standard IUMSS. ISO. 
Icontec. (2015). NTC ISO 14001:2015 Sistemas de gestión ambiental, requisitos con orientación para su uso. Icontec.

Icontec. (2015). NTC ISO 900o:2015 Sistemas de gestión de la calidad. Fundamentos y vocabulario. Icontec.

Icontec. (2015). NTC ISO 9001:2015 Sistemas de Gestión de la Calidad-Requisitos. Icontec.

Icontec. (2016). NTC ISO 13485:2016 Dispositivos médicos, gestión de la calidad, requisitos para propósitos regulatorios. Icontec.

Karapetrovic, S., y Jonker, J. (2003). Integration of Standardized Management Systems: Searching for a Recipe and Ingredients. Total Quality Management, 14(4), 451459. https://www.tandfonline.com/doi/abs/10.1080/1478336032000047264

Martínez Narváez, J. R. (2018). Propuesta Metodológica para la Integración de la NTC ISO 9001:2015 y NTC ISO 14001:2015 en el Sistema de Gestión de Calidad de Los Colegios Maristas de Colombia [tesis de maestría]. Universidad Santo Tomás.

Moreno, P. Restrepo, B. y Sanchez, P. (2020). Integración de las Normas NTCISO 14001:2005, NTCISO45001:2018 y NTCISO 13485:2016 y su Alineación a un Sistema de Gestión Integral Basado en la GTC 180:2008, en el Sector de Comercialización para Dispositivos Médicos en Colombia [tesis de maestría]. Universidad Santo Tomás. https://repository.usta.edu.co/handle/11634/27994

Moumen, M., y Aoufir, E. (2017). Quality, safety and environment management systems (QSE): analysis of empirical studies on integrated management systems (IMS). Journal of decision system, 26(3), 1-22. https://doi.org/10.1080/12460125.2017.1305648

Nunhes, V., Oliveira, O., y Bernardo, M. (2019). Guiding principles of integrated management systems: Towards unifying a starting point for researchers and practitioners. Journal of Cleaner Production, 210, 977-993. https://doi.org/10.1016/j.jclepro.2018.11.066

Nunhes, T., Motta, L., y Oliveira, O. (2017). Identification and analysis of the elements and functions integrable in integrated management systems. Journal of Cleaner Production, 142(4), 3225-3235. https://doi.org/10.1016/j.jclepro.2016.10.147 
Pullés, M. R., y Bataller Venta, M. (2016). Modelo de sistema integrado de gestión para una dirección de investigación medioambiental de Biocubafarma. CENIC, 46, 6-16. https://revista.cnic.cu/index.php/RevQuim/article/view/107

Wilkinson, G., y Dale, B. (1999). Integrated management systems: an examination of the concept and theory. The TQM Magazine, 11(2), 95-104.

https://www.emerald.com/insight/content/doi/10.1108/09544789910257280/full /html 